This item was submitted to Loughborough's Research Repository by the author.

Items in Figshare are protected by copyright, with all rights reserved, unless otherwise indicated.

\title{
Shubnikov-de Haas effect in multiband quasi-two-dimensional metals
}

PLEASE CITE THE PUBLISHED VERSION

PUBLISHER

(C) American Physical Society

VERSION

VoR (Version of Record)

LICENCE

CC BY-NC-ND 4.0

REPOSITORY RECORD

Thomas, I.O., V.V. Kabanov, and A.S. Alexandrov. 2019. "Shubnikov-de Haas Effect in Multiband Quasi-twodimensional Metals". figshare. https://hdl.handle.net/2134/4250. 
This item was submitted to Loughborough's Institutional Repository (https://dspace.lboro.ac.uk/) by the author and is made available under the following Creative Commons Licence conditions.

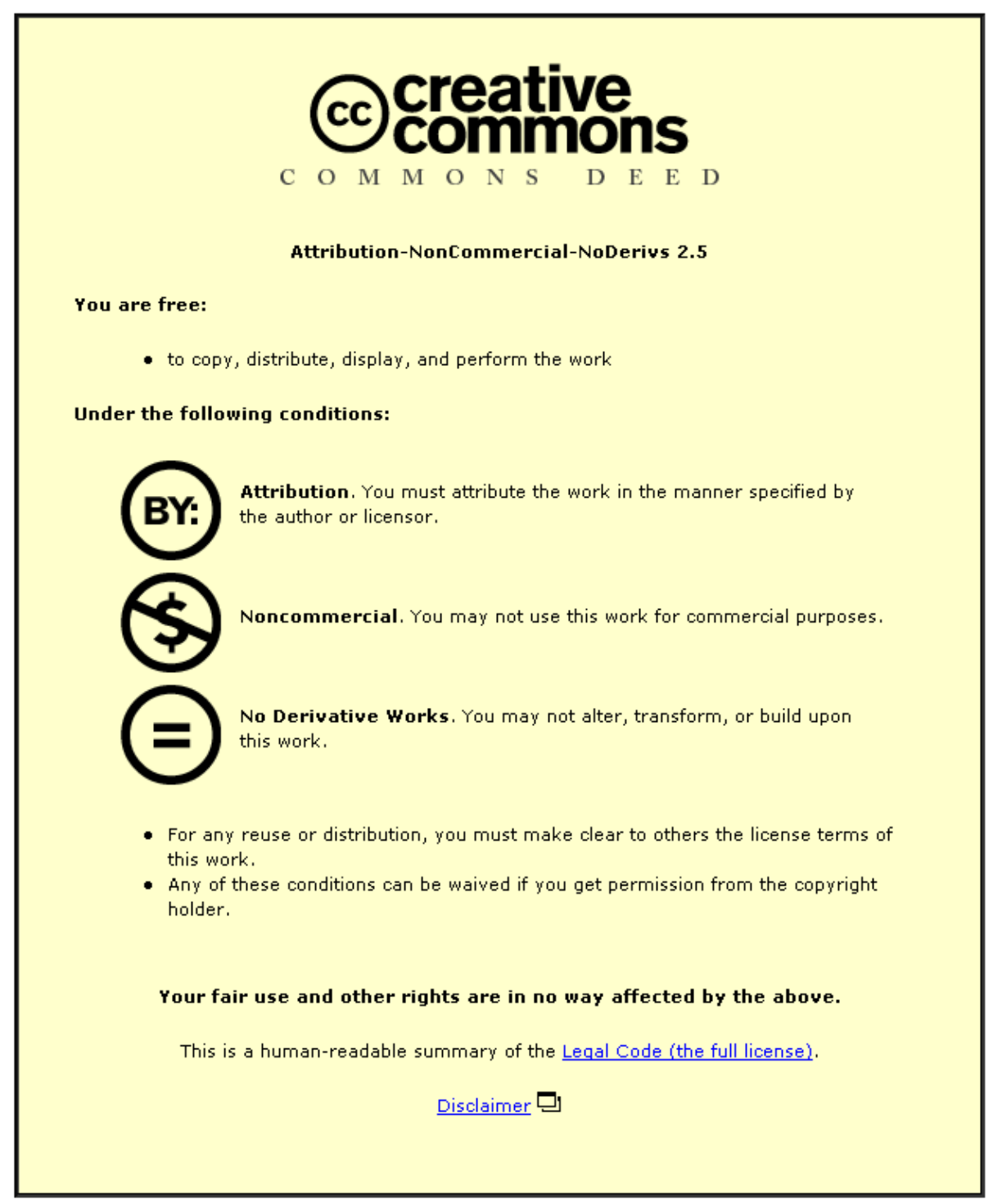

For the full text of this licence, please go to: http://creativecommons.org/licenses/by-nc-nd/2.5/ 


\title{
Shubnikov-de Haas effect in multiband quasi-two-dimensional metals
}

\author{
I. O. Thomas, ${ }^{1}$ V. V. Kabanov, ${ }^{2}$ and A. S. Alexandrov ${ }^{1}$ \\ ${ }^{1}$ Department of Physics, Loughborough University, Loughborough LE11 3TU, United Kingdom \\ ${ }^{2}$ Josef Stefan Institute, 1001 Ljubljana, Slovenia
}

(Received 1 November 2007; revised manuscript received 20 December 2007; published 28 February 2008)

\begin{abstract}
We analyze the behavior of the longitudinal conductivity $\sigma_{z z}$ in a field perpendicular to the highly conducting plane of a quasi-two-dimensional multiband metal in the case of a closed system where chemical potential oscillations may be observed compared with the case of an open system for various forms of scattering. In all but one case, we find that there are mixing frequencies present. However, they exhibit different qualitative behaviors, as befits their different origins, and in the case of interband scattering in an open system, may in fact be absent in the de Haas-van Alphen oscillations of that system.
\end{abstract}

DOI: 10.1103/PhysRevB.77.075434

PACS number(s): 73.63.-b, 72.15.Gd, 75.75.+a

\section{INTRODUCTION}

The use of magnetic quantum oscillations ${ }^{1}$ as a means of analyzing the Fermi surfaces of low-dimensional materials has received interest in recent years, particularly with respect to organic compounds such as the metallic varieties of the charge transfer salts bis(ethylenedithio)tetrathiafulvalene (BEDT-TFF) (see reviews by Singleton ${ }^{2}$ and Kartsovnik ${ }^{3}$ ) and also with respect to high temperature superconductors. ${ }^{4}$ One interesting feature of such systems is that the magnetic quantum oscillations can render measurable an apparent difference between the canonical (closed) and grand canonical (open) ensembles (in which the density of electrons $n_{e}$ and the chemical potential $\mu$ are fixed, respectively). In the former case, the chemical potential $\mu$ will oscillate, and this will have a noticeable effect on the behavior of the magnetic oscillations of a two-dimensional or quasi-two-dimensional system.

In two-dimensional multiband metals, frequency mixing in the de Haas-van Alphen effect resulting from chemical potential magneto-oscillations has been predicted, ${ }^{5}$ studied analytically and numerically, ${ }^{5-12}$ and observed experimentally $3,13,14$ in closed systems. The theoretical description has been generalized to the more realistic quasitwo-dimensional (quasi-2D) case. ${ }^{15}$ Recently, it was proposed that finite amplitudes due to mixing between extremal cross sections of the warped Fermi surface of a single-band closed quasi-2D system might also be observed. ${ }^{16}$ Unlike the previous examples, this particular mixing effect cannot be replicated by a magnetic breakdown. ${ }^{3,17}$

Attention has recently turned to the Shubnikov-de Haas $(\mathrm{SdH})$ oscillations of the longitudinal conductivity of quasi-2D single-band metals, with quantum transport theory being used by Champel and Mineev ${ }^{18}$ to examine the ultrahigh field 2D limit and by Grigoriev ${ }^{19}$ to examine the intermediate (quasi-2D) field limit. While much about the detailed physics of the former case remains obscure, ${ }^{20}$ an analysis of the latter case while taking into account scattering in the self-consistent Born approximation with $t \gg T_{D}$, where $T_{D}$ is the Dingle temperature and $t$ the interplane transfer integral, has resulted in a proposed explanation of a few oddities in the observed oscillations-namely, the existence of slow oscillations and the field dependent phase shifts of the beats of the conductivity.
In what follows, we examine the behavior of the longitudinal conductivity $\sigma_{z z}$ in a field perpendicular to the highly conducting plane of a quasi-2D multiband metal. We begin by deriving an expression for the conductivity of a two-band metal in the absence of any scattering or chemical potential oscillations in Sec. II A. We then (Sec. II B) derive an expression for the self-energy of a system in the Born approximation where there exists scattering between two bands, which we will have need of in some of our following calculations.

In the section following that, we calculate the effects of chemical potential oscillations and different forms of scattering on the system. First, in Sec. III A, we consider a closed system with a fixed relaxation time $\tau$, then in Sec. III B an open system with oscillations in $\tau$ arising from intraband scattering (this is merely a two-band generalization of Grigoriev's ${ }^{19}$ result), in Sec. III C a closed system with oscillations in $\tau$ arising from intraband scattering, in Sec. III D an open system with oscillations in $\tau$ arising from interband scattering, and, finally, in Sec. III E a closed system with oscillations in $\tau$ arising from interband scattering. In all cases other than the second, we discover some measure of frequency mixing, though the precise behavior of such mixing varies according to its origin. Interestingly, as argued at the beginning of Sec. III, one would probably not expect frequency mixing due to interband scattering to be very significant in the de Haas-van Alphen (dHvA) effect; that it is present in open systems in the $\mathrm{SdH}$ effect seems to indicate that one distinction between open and closed lowdimensional thermodynamic systems is slightly blurred in the case of conductivity.

\section{SHUBNIKOV-DE HAAS EFFECT WITH FIXED RELAXATION RATE}

\section{A. General expression for longitudinal conductivity}

A quasi-2D metal in a longitudinal magnetic field has the following energy spectrum: ${ }^{2,3}$

$$
\epsilon_{\alpha}\left(n, k_{z}\right)=\omega_{\alpha}(n+1 / 2)-2 t \cos \left(k_{z} d\right),
$$

where $\alpha$ labels the band, $d$ is the distance between layers, $n$ labels the Landau level, $k_{z}$ is the momentum in the perpen- 
dicular direction, and $\omega_{\alpha}=e B / m_{\alpha}$ is the cyclotron frequency, $m_{\alpha}$ being the effective mass for that band, and $\hbar=c=k_{B}=1$.

We may calculate the conductivity from the Kubo formula $^{21}$

$$
\sigma_{z z}=\frac{\pi e^{2}}{V} \int_{-\infty}^{\infty} d \xi\left[-n_{F}^{\prime}(\xi)\right] \overline{\operatorname{Tr}\left[\delta(\xi-H) \hat{v}_{z} \delta(\xi-H) \hat{v}_{z}\right]},
$$

where $\hat{v}_{z}$ is the velocity operator for the $z$ direction, $n_{F}^{\prime}$ is the derivative of the Fermi function, the trace is taken over all the single particle states $\beta=\left\{n, k_{z}, k_{y}, \alpha\right\} \equiv\{\gamma, \alpha\}$, the spin $V$ is the volume of the system, and the bar denotes the averaging over the random distribution of impurities in the sample. We may, if the impurities are pointlike, their concentration is low, and $t \gg T_{D}$, neglect the vertex corrections to the average and replace $\overline{\delta\left(\xi-\epsilon_{\beta}\right)}$ with $\Im G^{R}\left(\epsilon_{\beta}, \xi\right) / \pi$, where

$$
G^{R}\left(\epsilon_{\beta}, \xi\right)=\frac{1}{\xi-\epsilon_{\beta}-\Sigma_{\beta}(\xi)},
$$

is the retarded Green's function and $\Sigma_{\beta}(\xi)=L_{\beta}(\xi)-i \Delta_{\beta}(\xi)$ is the retarded self-energy $\left[L_{\beta}(\xi)\right.$ and $\Delta_{\beta}(\xi)$ being real], to obtain

$$
\sigma_{z z}=\frac{2 e^{2}}{V \pi} \int d \xi\left[-n_{F}^{\prime}(\xi)\right] \sum_{\alpha} \sum_{\gamma} v_{z \alpha, \gamma}^{2}\left[\Im G^{R}\left(\epsilon_{\alpha, \gamma}, \xi\right)\right]^{2} .
$$

This is simply the initial conductivity formula of Refs. 18 and 19, only now generalized so that it sums over multiple bands. Restricting our interest to the case of two bands with different masses $m_{1}$ and $m_{2}$, and since $t \gg T_{D}$, we may use the approximation $\Sigma_{\beta}(\xi) \approx \Sigma(\xi)$, making the calculation analytically tractable). Then, we may generalize the single-band result $^{18,19}$ as

$$
\begin{aligned}
\sigma_{z z}= & \frac{e^{2} N t d^{2}}{\pi} \int d \xi\left[-n_{F}^{\prime}(\xi)\right] \sum_{\alpha} \sum_{k=-\infty}^{\infty} \frac{(-1)^{k}}{k} J_{1}\left(\frac{4 \pi k t}{\omega_{\alpha}}\right) \\
& \times \exp \left(\frac{2 \pi i k \xi^{*}}{\omega_{\alpha}}\right)\left(\frac{1}{\Delta(\xi)}+\frac{2 \pi|k|}{\omega_{\alpha}}\right) R_{\alpha}(k, \xi) .
\end{aligned}
$$

Here, $J_{1}(x)$ is the first-order Bessel function, $\xi^{*}=\xi-\widetilde{L}(\xi)$, $\widetilde{L}(\xi)$ being the oscillating portion of $L(\xi), N=e B / 2 d \pi$, and

$$
R_{\alpha}(k, \xi)=\exp \left(\frac{-2 \pi \Delta(\xi)|k|}{\omega_{\alpha}}\right) .
$$

One should use the expansion $J_{1}(k x) / k=x / 2$ for the $k=0$ harmonic. This expression is relatively general but is not complete until the behavior of $\xi^{*}$ and $\Delta(\xi)$ is specified. In what remains of this subsection, we make use of a simple specification of their behavior in order to continue; more complex behaviors of these quantities are analyzed in Sec. III.

To begin with, let us assume that the quantum oscillations in the self-energy are suppressed, with the result that we can ignore the oscillatory contribution to $\xi^{*}$ and $\Delta(\xi)=(2 \tau)^{-1}$ $=\pi T_{D}$, where $\tau$ is the mean scattering time and $T_{D}$ is the associated Dingle temperature. This is justified in the presence of a field and size independent reservoir of states, ${ }^{15,18}$ for example, which suppresses both chemical potential oscillations and any oscillations in the self-energy. Having made this assumption, we integrate over $\xi$ [using the deltafunction-like behavior of $n_{F}^{\prime}(\xi)$ near the Fermi energy at small $T$ to obtain the first term, and $\int_{-\infty}^{\infty} \cos (a x) \cosh ^{-2}(x) d x$ $=a \pi / \sinh (a \pi / 2)$ to obtain the damping factor for the oscillatory terms] and so acquire

$$
\begin{aligned}
\sigma_{z z}= & \sigma\left[1+\sum_{\alpha} \frac{m_{\alpha}}{M} \sum_{k=1}^{\infty} \frac{\left(-R_{\alpha}\right)^{k}}{k} J_{1}\left(\frac{4 \pi k t}{\omega_{\alpha}}\right)\right. \\
& \left.\times\left(\frac{\omega_{\alpha}}{\pi t}+\frac{2 \pi k T_{D}}{t}\right) \cos \left(\frac{2 \pi k \mu}{\omega_{\alpha}}\right) \Theta\left(\frac{2 \pi^{2} k T}{\omega_{\alpha}}\right)\right],
\end{aligned}
$$

where $\quad \sigma=\left(e^{2} d t^{2} M\right) /\left(\pi^{2} T_{D}\right), \quad M=m_{1}+m_{2}, \quad R_{\alpha}$ $=\exp \left(-2 \pi^{2} T_{D} / \omega_{\alpha}\right)$ is the Dingle reduction factor, and $\Theta(y)=y / \sinh (y)$ is the usual Lifshitz-Kosevitch reduction factor. If $4 \pi t>\omega_{\alpha}$, we can use the asymptotic

$$
J_{1}\left(\frac{4 \pi k t}{\omega_{\alpha}}\right) \approx \sqrt{\frac{\omega_{\alpha}}{2 k \pi^{2} t}} \sin \left(\frac{4 \pi k t}{\omega_{\alpha}}-\frac{\pi}{4}\right),
$$

and so obtain

$$
\sigma_{z z}=\sigma\left[1+M^{-1} \sum_{\alpha} m_{\alpha} \sum_{k=1}^{\infty} A_{k}^{\alpha} \cos \left(\frac{2 \pi k \mu}{\omega_{\alpha}}\right) \Theta\left(\frac{2 \pi^{2} k T}{\omega_{\alpha}}\right)\right],
$$

where

$$
A_{k}^{\alpha}=\frac{\left(-R_{\alpha}\right)^{k}}{k} \sqrt{\frac{\omega_{\alpha}}{2 k \pi^{2} t}}\left(\frac{\omega_{\alpha}}{\pi t}+\frac{2 \pi k T_{D}}{t}\right) \sin \left(\frac{4 \pi k t}{\omega_{\alpha}}-\frac{\pi}{4}\right) .
$$

We can see then that the conductivity oscillates with two fundamental periods, one corresponding to the first band and one to the second. In the quasi-three-dimensional (quasi-3D) limit, one will observe the splitting of each fundamental in the Fourier transform of the conductivity into a pair of peaks (a low-frequency "neck" peak and a high-frequency "belly" peak), which is evidence of the slight warping of the Fermi surface such that there are two extremal orbits present. ${ }^{1}$

\section{B. Self-energy with interband scattering}

If scattering between bands is possible, then the selfenergy of the particles in any given band will contain contributions from all other bands in addition to that due to intraband scattering. In that case, in order to obtain the conductivity in the self-consistent Born approximation in the two-band case, we must include the impurity diagrams from both bands in $\Sigma_{\beta}(\xi),{ }^{22}$ 


$$
\Sigma^{R}(\xi)=W \sum_{\alpha} \sum_{\gamma} G^{R}\left(\epsilon_{\alpha, \gamma}, \xi\right),
$$

where $W$ is the square of the scattering amplitude, which is proportional to the impurity concentration and set equal to the same constant in every band for simplicity. It also includes a factor of 2 from the summation over the spin. Following Ref. 19, we find that

$$
\begin{aligned}
\sum_{\gamma} G^{R}\left(\epsilon_{\alpha, \gamma}, \xi\right) \\
=-\frac{V m_{\alpha}}{2 \pi d}\left[A(\xi)-2 \pi \sum_{k=1}^{\infty}(-1)^{k} J_{0}\left(\frac{4 \pi k t}{\omega_{\alpha}}\right)\right. \\
\quad \times \sin \left(\frac{2 \pi k \xi^{*}}{\omega_{\alpha}}\right) R_{\alpha}(k, \xi)+i \pi\left(1+2 \sum_{k=1}^{\infty}(-1)^{k} J_{0}\left(\frac{4 \pi k t}{\omega_{\alpha}}\right)\right. \\
\left.\left.\quad \times \cos \left(\frac{2 \pi k \xi^{*}}{\omega_{\alpha}}\right) R_{\alpha}(k, \xi)\right)\right] .
\end{aligned}
$$

From this, we can see that there is an oscillatory contribution from the real part of the self-energy that must be taken into account in our calculations and a slowly varying part $A(\xi)$ which may be ignored. Using our symmetric approximation [Eq. (11)] and setting $W V M / 2 \pi d=\pi T_{D}$ (as the average Dingle temperature is simply related to the average value of the imaginary part of the self-energy due to scattering), we may write the imaginary and oscillating real parts of the self-energy as follows:

$$
\begin{aligned}
\Delta(\xi)= & \pi T_{D}\left[1+2 M^{-1} \sum_{\alpha} m_{\alpha} \sum_{k=1}^{\infty}(-1)^{k} J_{0}\left(\frac{4 \pi k t}{\omega_{\alpha}}\right)\right. \\
& \left.\times \cos \left(\frac{2 \pi k \xi^{*}}{\omega_{\alpha}}\right) R_{\alpha}(k, \xi)\right], \\
\widetilde{L}(\xi)= & 2 \pi T_{D} M^{-1} \sum_{\alpha} m_{\alpha} \sum_{k=1}^{\infty}(-1)^{k} J_{0}\left(\frac{4 \pi k t}{\omega_{\alpha}}\right) \\
& \times \sin \left(\frac{2 \pi k \xi^{*}}{\omega_{\alpha}}\right) R_{\alpha}(k, \xi) .
\end{aligned}
$$

Equation (13) is a nonlinear equation for $\Delta(\xi)$, which can be solved approximately in the strong damping limit $R_{\alpha}(k, \xi) \ll 1$, as we shall see in Sec. III D.

\section{EFFECTS OF SCATTERING AND OSCILLATING $\mu$ ON THE CONDUCTIVITY}

In this section, we discuss the effects of chemical potential oscillations and of various kinds of scattering on the conductivity. Before we begin, however, we shall make some general observations regarding our results.

This analysis takes place in a region where there is strong damping of the amplitudes by the factor $R_{\alpha}$-we do not work in the region where the processes giving rise to mixing frequencies are at their strongest, and given that the effects we are interested in are of the second order in $R_{\alpha}$ [Eqs. (22),
(33), (44), and (46)], they may be quite small. However, it has been noted (by comparison to numerical results) that in the case of dHvA oscillations, ${ }^{9,16}$ a reasonable level of accuracy may be maintained even if one allows the Dingle damping factor to tend toward unity, assuming that one is in the appropriate limit regarding the behavior of $\mu$, so our results regarding mixing due to oscillations in $\mu$ may well still be valid in this limit.

It would also be surprising if our analysis of the systems where mixing due to interband scattering is present (Secs. III D and III E) were not even qualitatively correct (with regard to the presence of frequency mixing at the very least) outside of the region where $R_{\alpha} \ll 1$ since from Eq. (5) the $\mathrm{SdH}$ amplitudes contain a denominator $\Delta(\xi)$, which becomes more important as it becomes smaller, and $R_{\alpha}$, -which is dependent on $\Delta(\xi)$-approaches unity. Interestingly, this would not be the case with regard to dHvA oscillations. As can be seen from Eq. (68) of Ref. 23, $\Delta(\xi)$ enters only through the Dingle damping factor and the cosine function. Since $R_{\alpha}$ approaches 1 , the oscillating portions of $\Delta(\xi)$ become negligible, it is therefore doubtful that one would be able to observe frequency mixing due to scattering in the dHvA oscillations of any but the dirtiest systems. (This would entail the absence of measurable mixing frequencies in the dHvA oscillations of the system examined in Sec. III D, for example.)

With regard to the slow oscillations of the conductivity (which are found in all the systems analyzed below), it should be noted that it has been observed ${ }^{24}$ that macroscopic inhomogeneities in the sample increase the damping of the fast oscillations over and above that expected by simple scattering; this can be modeled by replacing the Dingle temperature in the $R_{\alpha}$ factors of those oscillations with a new, larger Dingle temperature $T_{D}^{*}$ that will enhance the damping of their amplitudes. In order to simplify our discussion though, we ignore this complication for now, though it should be taken into account in any physical measurement.

\section{A. Canonical ensemble: Oscillating $\mu$, fixed $\tau$}

In closed systems, the particle density is fixed, and we work in the canonical ensemble. As a result of this, the chemical potential $\mu$ of the system may oscillate as a function of the magnetic field. In three dimensions, these oscillations are negligible; however, as mentioned previously, in the case of multiband 2D and quasi-2D metals with two bands, one discovers additional harmonics corresponding to frequency mixing between bands. In this section, we discuss the effects of these oscillations on the conductivity of a system in which scattering effects are suppressed. This situation is physical if the oscillations in $\tau$ are suppressed due to a large amplitude of scattering from quantized bands to the reservoir, which is consistent with the existence of a small enough reservoir density of states for the oscillations in $\mu$ to remain significant.

Following Ref. 15, we divide the chemical potential into an oscillating part $\tilde{\mu}$ and a nonoscillating part $\mu_{0}$,

$$
\mu=\mu_{0}+\tilde{\mu},
$$

where 


$$
\tilde{\mu}=-\frac{1}{\rho}\left(\frac{\partial \widetilde{\Omega}}{\partial \mu}\right),
$$

with $\widetilde{\Omega}$ being the oscillatory portion of the thermodynamic potential $\Omega, \rho=\rho_{b g}+\Sigma_{\alpha} \rho_{\alpha}, \rho_{b g}$ being the unquantized background density of states, which we treat as being negligible, and $\rho_{\alpha}$ being the quantized density of states for the band $\alpha$. Using formula (14) of Ref. 15 and the 2D density of states $\rho_{\alpha}=m_{\alpha} / \pi$, we acquire

$$
\tilde{\mu}=-\sum_{\alpha} \sum_{k=1}^{\infty} B_{k}^{\alpha} \sin \left(\frac{2 \pi k\left(\mu_{0}+\tilde{\mu}\right)}{\omega_{\alpha}}\right) \Theta\left(\frac{2 \pi^{2} k T}{\omega_{\alpha}}\right),
$$

where

$$
\begin{aligned}
B_{k}^{\alpha} & =\frac{2 e B\left(R_{\alpha}\right)^{k}}{\pi M k} J_{0}\left(\frac{4 \pi k t}{\omega_{\alpha}}\right) \\
& \approx \frac{2 e B\left(R_{\alpha}\right)^{k}}{\pi M k} \sqrt{\frac{\omega_{\alpha}}{2 k \pi^{2} t}} \cos \left(\frac{4 \pi k t}{\omega_{\alpha}}-\frac{\pi}{4}\right),
\end{aligned}
$$

which follows from the use of the asymptotic

$$
J_{0}\left(\frac{4 \pi k t}{\omega_{\alpha}}\right) \approx \sqrt{\frac{\omega_{\alpha}}{2 k \pi^{2} t}} \cos \left(\frac{4 \pi k t}{\omega_{\alpha}}-\frac{\pi}{4}\right) .
$$

Let us assume that the system is strongly damped: $R_{\alpha} \ll 1$, and we keep only the oscillating terms of second order in $R_{\alpha}$ or less. It follows that $\tilde{\mu} / \omega_{\alpha} \ll 1$, so we may expand Eq. (9) in $\tilde{\mu}$ and approximate $\mu_{0}$ with $\mu$. We then insert Eq. (17), again approximating the argument of the sine function as $\mu \approx \mu_{0}$, and so obtain

$$
\sigma_{z z}=\sigma_{z z}^{o r d}+\sigma_{z z}^{m i x},
$$

where $\sigma_{z z}^{\text {ord }}$ represents the unmixed portion of the conductivity and $\sigma_{z z}^{\text {mix }}$ is the mixed portion. $\sigma_{z z}^{\text {ord }}$ is given by

$$
\begin{aligned}
\frac{\sigma_{z z}^{\text {ord }}}{\sigma}= & 1+M^{-1} \sum_{\alpha}\left(m_{\alpha} A_{1}^{\alpha} \Theta\left(\frac{2 \pi^{2} T}{\omega_{\alpha}}\right) \cos \left(\frac{2 \pi \mu}{\omega_{\alpha}}\right)\right. \\
& +\left[m_{\alpha} A_{2}^{\alpha} \Theta\left(\frac{4 \pi^{2} T}{\omega_{\alpha}}\right)+C_{\alpha \alpha} \Theta\left(\frac{2 \pi^{2} T}{\omega_{\alpha}}\right)^{2}\right] \cos \left(\frac{4 \pi \mu}{\omega_{\alpha}}\right) \\
& \left.-C_{\alpha \alpha} \Theta\left(\frac{2 \pi^{2} T}{\omega_{\alpha}}\right)^{2}\right)
\end{aligned}
$$

which shows that the oscillations in $\tilde{\mu}$ modify the second harmonics and also create additional slow oscillations due to the effect of the warping of the Fermi surface on the behavior of the scattering, ${ }^{24}$ as predicted for the single-band case by Grigoriev. ${ }^{19}$ This slow oscillation is not seen in dHvA oscillations (such as those of Ref. 16, for example); in those cases, one sees only the fast oscillations whose frequencies are determined by $t$ and $\mu$ together, with no slow oscillations whose frequencies are determined by $t$ alone.

The mixed component of the conductivity is given by

$$
\begin{aligned}
\frac{\sigma_{z z}^{m i x}}{\sigma}= & M^{-1}\left(C_{12}+C_{21}\right) \Theta\left(\frac{2 \pi^{2} T}{\omega_{1}}\right) \Theta\left(\frac{2 \pi^{2} T}{\omega_{2}}\right) \\
& \times\left[\cos \left(\frac{2 \pi \mu}{\omega_{+}}\right)-\cos \left(\frac{2 \pi \mu}{\omega_{-}}\right)\right],
\end{aligned}
$$

where

$$
\frac{1}{\omega_{ \pm}}=\frac{1}{\omega_{2}} \pm \frac{1}{\omega_{1}}
$$

and it is this term that gives rise to the frequency mixing induced by the chemical potential oscillations.

The amplitudes induced by the chemical potential oscillation are given by

$$
C_{\alpha \alpha}=\frac{2 e B m_{\alpha} a_{1, \alpha}}{M \pi^{2} t} R_{\alpha}^{2} \sin \left(2\left[\frac{4 \pi t}{\omega_{\alpha}}-\frac{\pi}{4}\right]\right),
$$

in the case where $\alpha=\alpha^{\prime}$, and

$$
C_{\alpha \alpha^{\prime}}=\frac{2 e B m_{\alpha} a_{1, \alpha}}{M \pi^{2} t}\left(\frac{m_{\alpha}}{m_{\alpha^{\prime}}}\right)^{1 / 2} R_{\alpha} R_{\alpha^{\prime}}\left[\cos \left(\frac{4 \pi t}{\omega_{+}}\right) \pm \sin \left(\frac{4 \pi t}{\omega_{-}}\right)\right],
$$

in the mixed $\left(\alpha \neq \alpha^{\prime}\right)$ case, where

$$
a_{r, \alpha}=\frac{1}{2}\left(\frac{\omega_{\alpha}}{r \pi t}+\frac{2 \pi T_{D}}{t}\right)
$$

and we take the positive sign in front of the sine when $\alpha^{\prime}=1$ and the negative when $\alpha^{\prime}=2$. One important feature of the mixing amplitudes in this case is that they are identical for both the additive and the subtractive mixing frequencies. This does not generally hold, as we shall see in what follows.

\section{B. Grand canonical ensemble: Intraband scattering}

Working in the self-consistent Born approximation, let us assume that there is no scattering between the bands and that we work in an open system described by the grand canonical ensemble. In this case, the only diagrams contributing to the self-energy of an electron in band $\alpha$ will be those corresponding to intraband scattering, and there are no chemical potential oscillations that could also result in a mixing of oscillation frequencies. This is the situation described by Grigoriev. ${ }^{19}$ Here, we simply generalize it to the case of multiple bands. Keeping all terms up to and including $O\left(R_{\alpha}^{2}\right)$, we obtain

$$
\begin{aligned}
\sigma_{z z}= & \sigma\left[1+M^{-1} \sum_{\alpha} m_{\alpha}\left[D_{1}^{\alpha} \Theta\left(\frac{2 \pi^{2} T}{\omega_{\alpha}}\right) \cos \left(\frac{2 \pi \mu}{\omega_{\alpha}}\right)\right.\right. \\
& \left.\left.+D_{2}^{\alpha} \Theta\left(\frac{4 \pi^{2} T}{\omega_{\alpha}}\right) \cos \left(\frac{4 \pi \mu}{\omega_{\alpha}}\right)+D_{S}^{\alpha}\right]\right]
\end{aligned}
$$

where 


$$
\begin{aligned}
D_{1}^{\alpha}= & 2 \sqrt{\frac{\omega_{\alpha}}{2 \pi^{2} t}\left[1+\left(a_{1, \alpha}\right)^{2}\right]} R_{\alpha} \cos \left(\frac{4 \pi t}{\omega_{\alpha}}-\frac{\pi}{4}+\phi_{1, \alpha}\right) \\
D_{2}^{\alpha}= & \frac{4 T_{D}}{t} \sqrt{1+\left(a_{1, \alpha}\right)^{2}}\left(R_{\alpha}\right)^{2} \cos \left(\frac{4 \pi t}{\omega_{\alpha}}-\frac{\pi}{4}\right) \\
& \times \cos \left(\frac{4 \pi t}{\omega_{\alpha}}-\frac{\pi}{4}+\phi_{1, \alpha}\right)+\left(R_{\alpha}\right)^{2} \sqrt{\frac{\omega_{\alpha}}{\pi^{2} t}\left[1+\left(a_{2, \alpha}\right)^{2}\right]} \\
& \times \cos \left(\frac{8 \pi t}{\omega_{\alpha}}-\frac{\pi}{4}+\phi_{2, \alpha}\right)+D_{S}^{\alpha}, \\
D_{S}^{\alpha}= & \frac{\omega_{\alpha}\left(R_{\alpha}\right)^{2}}{2 \pi^{2} t} \\
& \times\left[\sqrt{1+\left(\frac{\omega_{\alpha}}{2 \pi t}\right)^{2}} \cos \left(2\left[\frac{4 \pi t}{\omega_{\alpha}}-\frac{\pi}{4}+\phi_{S \alpha}\right]\right)+1\right] \\
& \phi_{r, \alpha}=\arctan \left(a_{r, \alpha}\right), \quad \phi_{S, \alpha}=\arctan \left(\frac{\omega_{\alpha}}{2 \pi t}\right)
\end{aligned}
$$

and $a_{r, \alpha}$ is given by Eq. (26).

\section{Canonical ensemble: Oscillating $\mu$, Intraband scattering}

When the system is closed, we must take into account the effects of the oscillations in $\mu$ as well as that of scattering. We can do this by applying the procedure outlined in Sec. III A to Eq. (27), assuming that $R_{\alpha} \ll 1$.

Having performed the expansion in terms of $\tilde{\mu}$, we may write our result as

$$
\sigma_{z z}=\sigma_{z z}^{o r d}+\sigma_{z z}^{m i x}
$$

where

$$
\begin{aligned}
\frac{\sigma_{z z}^{\text {ord }}}{\sigma}= & 1+M^{-1} \sum_{\alpha} m_{\alpha}\left(D_{1}^{\alpha} \Theta\left(\frac{2 \pi^{2} T}{\omega_{\alpha}}\right) \cos \left(\frac{2 \pi \mu}{\omega_{\alpha}}\right)\right. \\
& +\left[D_{2}^{\alpha} \Theta\left(\frac{4 \pi^{2} T}{\omega_{\alpha}}\right)-\mathcal{C}_{\alpha \alpha} \Theta\left(\frac{2 \pi^{2} T}{\omega_{\alpha}}\right)^{2}\right] \cos \left(\frac{4 \pi \mu}{\omega_{\alpha}}\right)+D_{S}^{\alpha} \\
& \left.+\mathcal{C}_{\alpha \alpha} \Theta\left(\frac{2 \pi^{2} T}{\omega_{\alpha}}\right)^{2}\right)
\end{aligned}
$$

and

$$
\begin{aligned}
\frac{\sigma_{z z}^{\text {mix }}}{\sigma}= & M^{-1}\left(m_{1} \mathcal{C}_{12}+m_{2} \mathcal{C}_{21}\right) \Theta\left(\frac{2 \pi^{2} T}{\omega_{1}}\right) \Theta\left(\frac{2 \pi^{2} T}{\omega_{2}}\right) \\
& \times\left[\cos \left(\frac{2 \pi \mu}{\omega_{-}}\right)-\cos \left(\frac{2 \pi \mu}{\omega_{+}}\right)\right] .
\end{aligned}
$$

The amplitudes due to the chemical potential oscillations are given by

$$
\begin{aligned}
\mathcal{C}_{\alpha \alpha}= & \frac{e B R_{\alpha}^{2}}{M \pi^{2} t} \sqrt{1+\left(a_{r, \alpha}\right)^{2}} \\
& \times\left[\cos \left(\phi_{1, \alpha}\right)+\cos \left(2\left[\frac{4 \pi t}{\omega_{\alpha}}-\frac{\pi}{4}+\frac{\phi_{1, \alpha}}{2}\right]\right)\right]
\end{aligned}
$$

when $\alpha=\alpha^{\prime}$ and by the following when $\alpha \neq \alpha^{\prime}$ :

$$
\begin{aligned}
\mathcal{C}_{\alpha \alpha^{\prime}}= & \frac{e B R_{\alpha} R_{\alpha^{\prime}}}{M \pi^{2} t}\left(\frac{m_{\alpha}}{m_{\alpha^{\prime}}}\right)^{1 / 2} \sqrt{1+\left(a_{r, \alpha}\right)^{2}} \\
& \times\left[\cos \left(\frac{4 \pi t}{\omega_{-}} \pm \phi_{1, \alpha}\right)-\sin \left(\frac{4 \pi t}{\omega_{+}}+\phi_{1, \alpha}\right)\right],
\end{aligned}
$$

where the argument of the cosine function contains a " + " if $\alpha=2$ and a "-" if $\alpha=1$.

In this case, we find that we have two terms contributing to the slow oscillations, one of which is temperature dependent, and that (as one might expect from Sec. III A) we have additional frequencies due to the mixing of bands by the oscillations in the chemical potential.

\section{Grand canonical ensemble: Interband scattering}

In order to proceed with our calculation of the effects of interband scattering on the behavior of the system in the grand canonical ensemble, we expand Eq. (13) in the strong damping limit $R_{\alpha}(k, \xi) \ll 1$, where we make the approximation

$$
\begin{aligned}
R_{\alpha}(1, \xi) \approx & R_{\alpha}\left[1+\frac{4 \pi^{2} T_{D}}{\omega_{\alpha} M} \sum_{\alpha^{\prime}} m_{\alpha^{\prime}} J_{0}\left(\frac{4 \pi t}{\omega_{\alpha^{\prime}}}\right) \cos \left(\frac{2 \pi \xi}{\omega_{\alpha^{\prime}}}\right) R_{\alpha^{\prime}}\right] \\
& R_{\alpha}(2, \xi) \approx R_{\alpha}^{2} .
\end{aligned}
$$

We can then expand out the cosine terms for small $\tilde{L}(\xi)$ and find that for $k=1$,

$$
\begin{aligned}
& \cos \left(\frac{2 \pi \xi^{*}}{\omega_{\alpha}}\right) R_{\alpha}(1, \xi) \\
& \approx \cos \left(\frac{2 \pi \xi}{\omega_{\alpha}}\right) R_{\alpha}+\frac{4 \pi^{2} T_{D}}{\omega_{\alpha} M}\left(R_{\alpha}\right)^{2} m_{\alpha} J_{0}\left(\frac{4 \pi t}{\omega_{\alpha}}\right) \cos \left(\frac{4 \pi \xi}{\omega_{\alpha}}\right) \\
& \quad+\frac{4 \pi^{2} T_{D}}{\omega_{\alpha} M} R_{\alpha} R_{\bar{\alpha}} m_{\bar{\alpha}} J_{0}\left(\frac{4 \pi t}{\omega_{\bar{\alpha}}}\right) \cos \left(\frac{2 \pi \xi}{\omega^{+}}\right)
\end{aligned}
$$

where if $\alpha=1,2$, then $\bar{\alpha}=2,1$, and that for $k=2$,

$$
\cos \left(\frac{4 \pi \xi^{*}}{\omega_{\alpha}}\right) R_{\alpha}(2, \xi) \approx \cos \left(\frac{4 \pi \xi}{\omega_{\alpha}}\right) R_{\alpha}^{2}
$$

The conductivity in this approximation is given by [after the substitution of Eq. (13) into Eq. (5)] 


$$
\begin{aligned}
\sigma_{z z}= & \sum_{\alpha} \sigma_{\alpha} \int d \xi\left[-n_{F}^{\prime}(\xi)\right]\left[1-\frac{\omega_{\alpha}}{\pi t} J_{1}\left(\frac{4 \pi t}{\omega_{\alpha}}\right) \cos \left(\frac{2 \pi \xi^{*}}{\omega_{\alpha}}\right) R_{\alpha}(1, \xi)+\frac{\omega_{\alpha}}{2 \pi t} J_{1}\left(\frac{8 \pi t}{\omega_{\alpha}}\right) \cos \left(\frac{4 \pi \xi^{*}}{\omega_{\alpha}}\right) R_{\alpha}(2, \xi)\right] \\
& \times\left[1+2 M^{-1} \sum_{\alpha^{\prime}} R_{\alpha^{\prime}}(1, \xi) m_{\alpha^{\prime}} J_{0}\left(\frac{4 \pi t}{\omega_{\alpha^{\prime}}}\right) \cos \left(\frac{2 \pi \xi^{*}}{\omega_{\alpha^{\prime}}}\right)-2 M^{-1} \sum_{\alpha^{\prime}} R_{\alpha^{\prime}}(2, \xi) m_{\alpha^{\prime}} J_{0}\left(\frac{8 \pi t}{\omega_{\alpha^{\prime}}}\right) \cos \left(\frac{4 \pi \xi^{*}}{\omega_{\alpha^{\prime}}}\right)\right. \\
& \left.+\left(2 M^{-1} \sum_{\alpha^{\prime}} R_{\alpha^{\prime}}(1, \xi) m_{\alpha^{\prime}} J_{0}\left(\frac{4 \pi t}{\omega_{\alpha^{\prime}}}\right) \cos \left(\frac{2 \pi \xi^{*}}{\omega_{\alpha^{\prime}}}\right)\right)^{2}\right] \\
& +\frac{2 \pi^{2} T_{D}}{\omega_{\alpha}}\left[-J_{1}\left(\frac{4 \pi t}{\omega_{\alpha}}\right) \cos \left(\frac{2 \pi \xi^{*}}{\omega_{\alpha}}\right) R_{\alpha}(1, \xi)+J_{1}\left(\frac{8 \pi t}{\omega_{\alpha}}\right) \cos \left(\frac{4 \pi \xi^{*}}{\omega_{\alpha}}\right) R_{\alpha}(2, \xi)\right],
\end{aligned}
$$

where $\sigma_{\alpha}=\left(e^{2} d t^{2} m_{\alpha}\right) /\left(\pi^{2} T_{D}\right)$.

Substituting Eqs. (36)-(38) into Eq. (39), integrating over $\xi$, replacing the Bessel functions with their asymptotics, and gathering all the terms together, we finally obtain

$$
\begin{aligned}
\sigma_{z z}= & \sigma\left[1+\sum_{\alpha}\left(\mathcal{D}_{1}^{\alpha} \cos \left(\frac{2 \pi \mu}{\omega_{\alpha}}\right) \Theta\left(\frac{2 \pi^{2} T}{\omega_{\alpha}}\right)\right.\right. \\
& \left.+\mathcal{D}_{2}^{\alpha} \cos \left(\frac{4 \pi \mu}{\omega_{\alpha}}\right) \Theta\left(\frac{4 \pi^{2} T}{\omega_{\alpha}}\right)+\mathcal{D}_{S}^{\alpha}\right) \\
& +\mathcal{D}_{12}^{+} \cos \left(\frac{2 \pi \mu}{\omega_{+}}\right) \Theta\left(\frac{2 \pi^{2} T}{\omega_{+}}\right) \\
& \left.+\mathcal{D}_{12}^{-} \cos \left(\frac{2 \pi \mu}{\omega_{-}}\right) \Theta\left(\frac{2 \pi^{2} T}{\omega_{-}}\right)\right] .
\end{aligned}
$$

Here, the unmixed amplitudes are

$$
\begin{aligned}
\mathcal{D}_{1}^{\alpha}= & 2 \frac{m_{\alpha}^{1 / 2}}{M} \sqrt{\frac{e B}{2 \pi^{2} t}\left[1+\left(a_{1, \alpha}\right)^{2}\right]} R_{\alpha} \cos \left(\frac{4 \pi t}{\omega_{\alpha}}-\frac{\pi}{4}+\phi_{1, \alpha}\right), \\
\mathcal{D}_{2}^{\alpha}= & \frac{\left(R_{\alpha}\right)^{2} m_{\alpha}}{M}\left[\frac{4 m_{\alpha} T_{D}}{M t} \sqrt{1+\left(a_{1, \alpha}\right)^{2}}\right. \\
& \times \cos \left(\frac{4 \pi t}{\omega_{\alpha}}-\frac{\pi}{4}\right) \cos \left(\frac{4 \pi t}{\omega_{\alpha}}-\frac{\pi}{4}+\phi_{1, \alpha}\right) \\
& \left.+2 \sqrt{\frac{\omega_{\alpha}}{4 \pi^{2} t}\left[1+\left(a_{2, \alpha}\right)^{2}\right]} \cos \left(\frac{8 \pi t}{\omega_{\alpha}}-\frac{\pi}{4}+\phi_{2, \alpha}\right)\right]+\mathcal{D}_{S}^{\alpha},
\end{aligned}
$$

and

$$
\begin{aligned}
\mathcal{D}_{S}^{\alpha}= & \frac{e B\left(R_{\alpha}\right)^{2} m_{\alpha}}{2 \pi^{2} M^{2} t}\left[\sqrt{1+\left(\frac{\omega_{\alpha}}{2 \pi t}\right)^{2}}\right. \\
& \left.\times \cos \left(2\left[\frac{4 \pi t}{\omega_{\alpha}}-\frac{\pi}{4}+\phi_{S \alpha}\right]\right)+1\right] .
\end{aligned}
$$

As in the case of oscillating $\mu$, we observe mixed frequencies as well,

$$
\begin{aligned}
\mathcal{D}_{12}^{+}= & \frac{e B R_{1} R_{2} \sqrt{m_{1} m_{2}}}{M^{2} t \pi^{2}}\left(1+\frac{2 \pi^{2} T_{D}}{\omega_{+}}\right)\left[\sqrt{1+\left(y^{-}\right)^{2}}\right. \\
& \left.\times \cos \left(\frac{4 \pi t}{\omega_{-}}+\phi_{y^{-}}\right)+\sqrt{1+\left(y^{+}\right)^{2}} \sin \left(\frac{4 \pi t}{\omega_{+}}+\phi_{y^{+}}\right)\right],
\end{aligned}
$$

where

$$
\begin{aligned}
y^{ \pm}= & \left(\frac{\left(\omega_{2} \pm \omega_{1}\right)}{4 \pi t}+2 \pi^{2} T_{D}\left[\frac{a_{1,2}}{\omega_{2}} \pm \frac{a_{1,1}}{\omega_{1}}\right]\right) \\
& \times\left(1+\frac{2 \pi^{2} T_{D}}{\omega_{+}}\right)^{-1}, \quad \phi_{y^{ \pm}}=\arctan \left(y^{ \pm}\right),
\end{aligned}
$$

and

$$
\begin{aligned}
\mathcal{D}_{12}^{-}= & \frac{e B R_{1} R_{2} \sqrt{m_{1} m_{2}}}{M^{2} t \pi^{2}}\left[\sqrt{1+\left(q^{-}\right)^{2}} \cos \left(\frac{4 \pi t}{\omega_{-}}+\phi_{q^{-}}\right)\right. \\
& \left.+\sqrt{1+\left(q^{+}\right)^{2}} \sin \left(\frac{4 \pi t}{\omega_{+}}+\phi_{q^{+}}\right)\right],
\end{aligned}
$$

where

$$
q^{ \pm}=\frac{\left(\omega_{2} \pm \omega_{1}\right)}{4 \pi t}, \quad \phi_{q^{ \pm}}=\arctan \left(q^{ \pm}\right) .
$$

As before, the values of $a_{r, \alpha}$ and $\phi_{r, \alpha}$ and $\phi_{S \alpha}$ are given by Eqs. (26) and (30), respectively.

\section{E. Canonical ensemble: Oscillating $\mu$, interband scattering}

Let us now treat the above system as though it were closed, and so allow $\mu$ to oscillate. The only terms of interest to us will come from the expansion of the terms proportional to $\mathcal{D}_{1}^{\alpha}$. From an examination of Eqs. (28) and (41), we can see that

$$
\mathcal{D}_{1}^{\alpha}=\frac{m_{\alpha}}{M} D_{1}^{\alpha},
$$

and it follows that the amplitudes due to the chemical potential oscillations are

$$
\overline{\mathcal{C}}_{\alpha \alpha}=\frac{m_{\alpha}}{M} \mathcal{C}_{\alpha \alpha}, \quad \overline{\mathcal{C}}_{\alpha \alpha^{\prime}}=\frac{m_{\alpha}}{M} \mathcal{C}_{\alpha \alpha^{\prime}}
$$

and that the expression for the conductivity is 


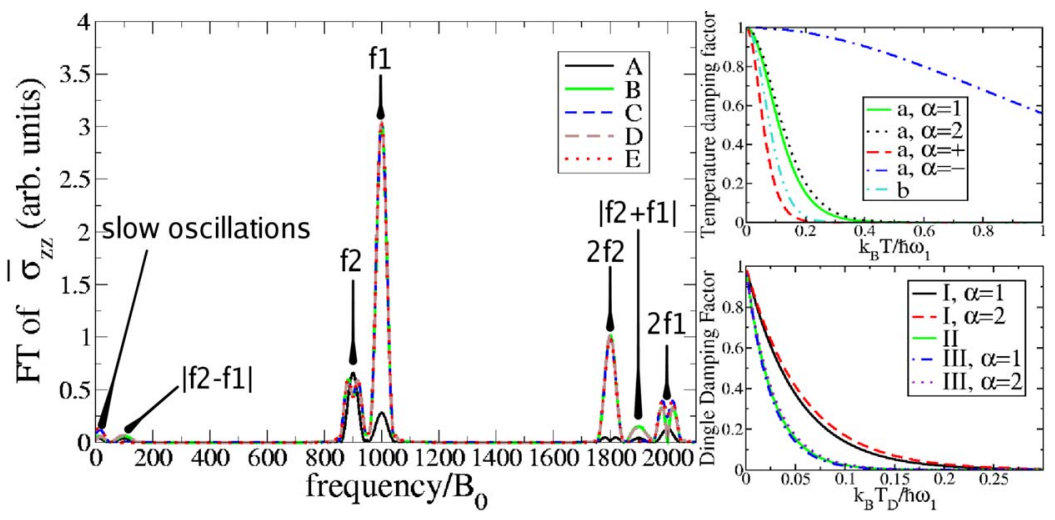

FIG. 1. (Color online) A graph showing the Fourier transform of the $\mathrm{SdH}$ oscillations from $0.9 \leqslant B_{0} / B \leqslant 0.95$ where the frequencies are given in terms of $B / B_{0}$, alongside plots of how the damping factors evolve as their respective temperatures are increased. Due to the small size of the window, the slow oscillations are poorly resolved. The $y$ axis has been rescaled by a factor of 100 . The following parameters are used: $\mu=500 t,\left(\omega_{1} / \omega_{2}\right)=\left(m_{2} / m_{1}\right)=0.9, k_{B} T_{D}=0.026 t$, and $k_{B} T=0.00005 t$, setting our unit of measurement to be $B_{0}=m_{1} t / 2 \hbar e$, which is around $45 \mathrm{~T}$ if $t=0.01 \mathrm{eV}$ and $m_{1}=m_{e}$. The legend in the Fourier transform plots should be interpreted as follows: A—closed system, fixed $\tau$; B-open system, interband scattering; $\mathrm{C}$-closed system, intraband scattering; D—closed system, interband scattering; E—open system, intraband scattering. The legend in the temperature damping plots should be interpreted as a-value of $\Theta\left(2 \pi^{2} T / \omega_{\alpha}\right)$; b-value of $\Theta\left(2 \pi^{2} T / \omega_{1}\right) \Theta\left(2 \pi^{2} T / \omega_{2}\right)$. The legend in the dingle damping plots should be interpreted as: I-value of $R_{\alpha}$; II-value of $R_{1} R_{2}$; III-value of $R_{\alpha}^{2}$.

$$
\begin{aligned}
\sigma_{z z}= & \sigma\left[1+\sum_{\alpha}\left(\mathcal{D}_{1}^{\alpha} \cos \left(\frac{2 \pi \mu}{\omega_{\alpha}}\right) \Theta\left(\frac{2 \pi^{2} T}{\omega_{\alpha}}\right)+\left[\mathcal{D}_{2}^{\alpha} \Theta\left(\frac{4 \pi^{2} T}{\omega_{\alpha}}\right)-\overline{\mathcal{C}}_{\alpha \alpha} \Theta\left(\frac{2 \pi^{2} T}{\omega_{\alpha}}\right)^{2}\right] \cos \left(\frac{4 \pi \mu}{\omega_{\alpha}}\right)+\mathcal{D}_{S}^{\alpha}+\overline{\mathcal{C}}_{\alpha \alpha} \Theta\left(\frac{2 \pi^{2} T}{\omega_{\alpha}}\right)^{2}\right)\right. \\
& +\left[\mathcal{D}_{12}^{+} \Theta\left(\frac{2 \pi^{2} T}{\omega_{+}}\right)-\left(\overline{\mathcal{C}}_{12}+\overline{\mathcal{C}}_{21}\right) \Theta\left(\frac{2 \pi^{2} T}{\omega_{1}}\right) \Theta\left(\frac{2 \pi^{2} T}{\omega_{2}}\right)\right] \cos \left(\frac{2 \pi \mu}{\omega_{+}}\right) \\
& \left.+\left[\mathcal{D}_{12}^{-} \Theta\left(\frac{2 \pi^{2} T}{\omega_{-}}\right)+\left(\overline{\mathcal{C}}_{12}+\overline{\mathcal{C}}_{21}\right) \Theta\left(\frac{2 \pi^{2} T}{\omega_{1}}\right) \Theta\left(\frac{2 \pi^{2} T}{\omega_{2}}\right)\right] \cos \left(\frac{2 \pi \mu}{\omega_{-}}\right)\right] .
\end{aligned}
$$

In this case, all the second order terms are modified by the chemical potential oscillations.

\section{DISCUSSION}

Figures 1-4 display some illustrative Fourier transforms of the oscillatory components $\bar{\sigma}_{z z}$ of $\left(\sigma_{z z}-\sigma\right) / \sigma$ given by the expressions in the previous sections. The inclusion of plots of the behavior of the Dingle and temperature reduction fac- tors in the figures should facilitate the extrapolation of the results given here to regimes where $R_{\alpha} \ll 1$, where our analytic calculations are more valid; however, it would be surprising if the qualitative elements of our results were not preserved even at values of $R_{\alpha}$ near unity. For the purposes of reference to a real material the measurements by Cole et $a l{ }^{25}$ of the effective mass in the two-band system of GaAs$(\mathrm{Ga}, \mathrm{Al})$ As heterojunctions indicate a ratio of masses somewhere in the region $0.3 \lesssim\left(m_{2} / m_{1}\right) \lesssim 0.45$.

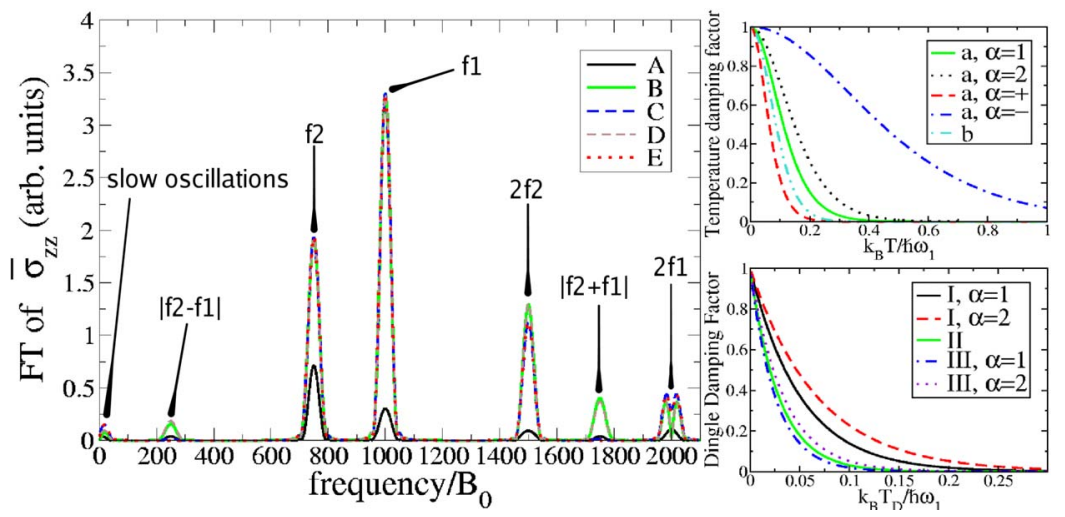

FIG. 2. (Color online) A graph showing the Fourier transform of the $\mathrm{SdH}$ oscillations from $0.9 \leqslant B_{0} / B \leqslant 0.95$ with $\left(\omega_{1} / \omega_{2}\right)=\left(m_{2} / m_{1}\right)=0.75$, alongside plots of how the damping factors evolve as their respective temperatures are increased. Due to the small size of the window, the slow oscillations are poorly resolved. The $y$ axis has been rescaled by a factor of 100. Except as otherwise noted, all parameters and legends are identical to those in Fig. 1. 


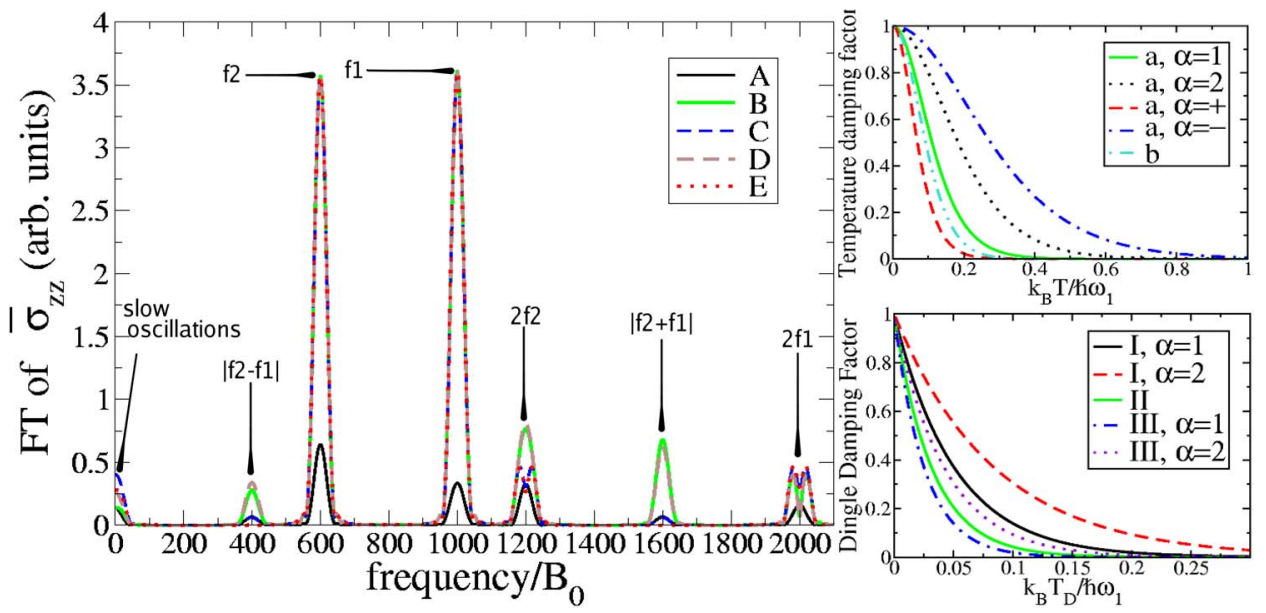

FIG. 3. (Color online) A graph showing the Fourier transform of the $\mathrm{SdH}$ oscillations from $0.9 \leqslant B_{0} / B \leqslant 0.95$ with $\left(\omega_{1} / \omega_{2}\right)$ $=\left(m_{2} / m_{1}\right)=0.6$, alongside plots of how the damping factors evolve as their respective temperatures are increased. Due to the small size of the window, the slow oscillations are poorly resolved. The $y$ axis has been rescaled by a factor of 100. Except as otherwise noted, all parameters and legends are identical to those in Fig. 1.
Note that in order to clean up the signal and remove spurious (ringing) due to the finite window size, the data were passed through a Hanning window prior to the operation of the numerical Fourier transform (see, for example, Ref. 26).

Details of the mixing frequencies are shown in Figs. 5 and 6. Perhaps the most obvious and interesting feature is that the signature of the two kinds of mixing is not the same: The amplitudes of the $\left|f_{2}+f_{1}\right|$ and $\left|f_{2}-f_{1}\right|$ mixings are identical in the cases where the mixing is due to only oscillations in $\mu$, but in cases where mixing through interband scattering is present, the $\left|f_{2}-f_{1}\right|$ mixing amplitude is less than that of the $\left|f_{2}+f_{1}\right|$ mixings (the exception is case $\mathrm{C}$ at a ratio of 0.9 , most likely due to the small size of its amplitude and its being interfered with by neighboring peaks). In general, the amplitude of the mixing peaks grows more pronounced as the two bands become less similar (that is, as $m_{2}$ becomes smaller than $m_{1}$ ), at least for these values of $m_{2}$, apart from case $\mathrm{A}$ at a ratio of 0.4 , where a splitting of the peaks reduces their size. It should also be noted from the structure of the Lifschitz-Kosevich factors in the mixing terms of Eqs. (20) and (40) that the amplitudes behave differently with respect to the temperature and that this provides a further method of distinguishing between the two sources of oscillation (see Ref. 27 for the relevance of this point in the context of the theory of magnetic breakdown).

Our calculations can also be applied to quasi-threedimensional metals, where $t$ is less than or comparable to $\mu$. Figure 7 shows the Fourier transform of $\bar{\sigma}_{z z}$ when $\mu=5 t, \quad\left(\omega_{1} / \omega_{2}\right)=\left(m_{2} / m_{1}\right)=0.75, \quad k_{B} T=2.5 \times 10^{-7} t, \quad B_{0}$ $=m_{1} t /(200 \hbar e)$, and we have set $T_{D}$ to be zero, in order to clarify the harmonic behavior of the amplitudes. In this case, our results correspond to a Fourier series truncated after the second harmonics of each band, and we can see that the mixing effects due to scattering or chemical potential oscillations are suppressed in this limit. We can observe that the ratio between the first and second harmonics is $\approx 2^{1 / 2}$ in the cases where we have scattering and $\approx 2^{3 / 2}$ in the case of oscillating $\mu$ alone. This difference is due to our neglect of the contribution from scattering to the oscillations in the latter case, which, as we can see from a comparison of the amplitudes in the graphs, is the dominant source of oscillations in three dimensions (as one might expect from the results of calculations in 3D metals ${ }^{28}$ ). Naturally, at finite temperatures, the amplitudes will decay correspondingly more quickly due to the effect of the temperature and Dingle damping factors, which can be extrapolated from their respective plots displayed in the figure.

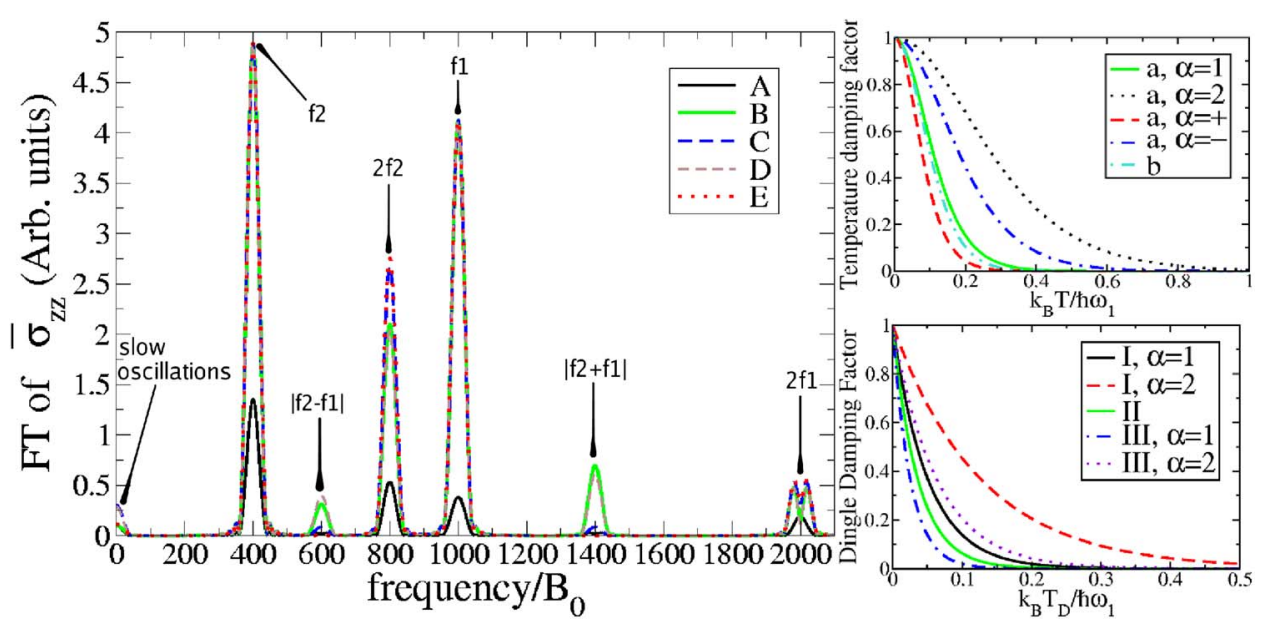

FIG. 4. (Color online) A graph showing the Fourier transform of the $\mathrm{SdH}$ oscillations from $0.9 \leqslant B_{0} / B \leqslant 0.95$ with $\left(\omega_{1} / \omega_{2}\right)$ $=\left(m_{2} / m_{1}\right)=0.4$, alongside plots of how the damping factors evolve as their respective temperatures are increased. Due to the small size of the window, the slow oscillations are poorly resolved. The $y$ axis has been rescaled by a factor of 100. Except as otherwise noted, all parameters and legends are identical to those in Fig. 1. 


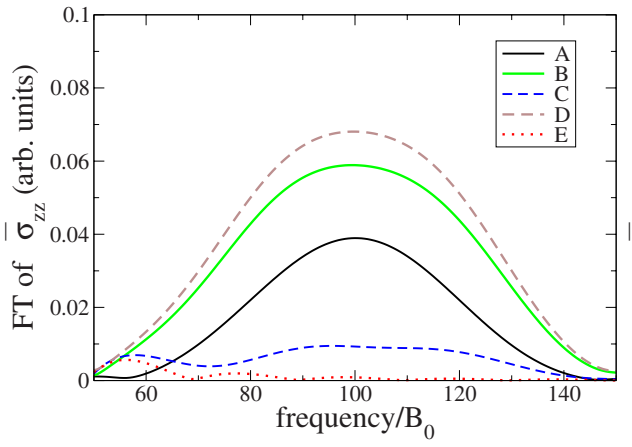

(a)

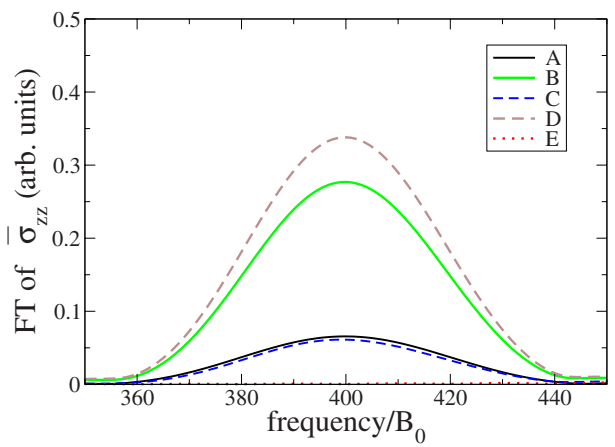

(c)

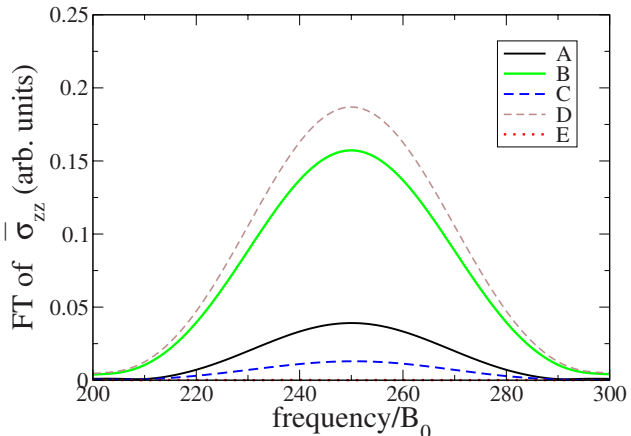

(b)

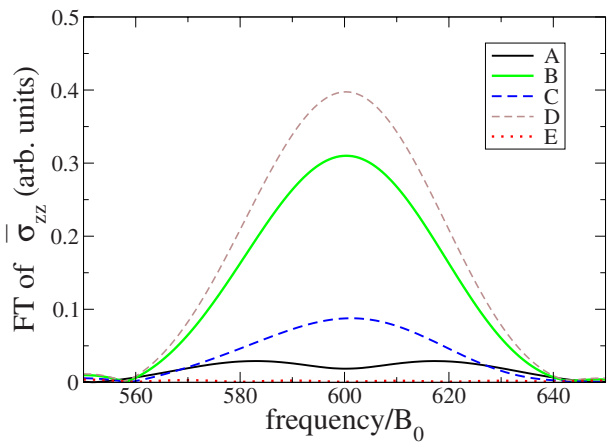

(d)
FIG. 5. (Color online) Figures showing the details of the $\left|f_{2}-f_{1}\right|$ amplitudes of $\left(m_{2} / m_{1}\right)$ values of (a) 0.9 , (b) 0.75 , (c) 0.6 , and (d) 0.4 .

\section{CONCLUSION}

We have examined possible sources of frequency mixing in the SdH oscillations of multiband quasi-2D metals in the canonical and grand canonical metallic ensembles in the intermediate values of the field where $4 \pi t>\omega_{\alpha}$. We considered a closed system with a fixed relaxation time $\tau$, an open system with oscillations in $\tau$ arising from intraband scattering, a closed system with oscillations in $\tau$ arising from intraband scattering, an open system with oscillations in $\tau$ arising from interband scattering, and, lastly, a closed system with interband scattering with oscillations in $\tau$ arising from interband scattering.

In all cases other than the second, we discover some

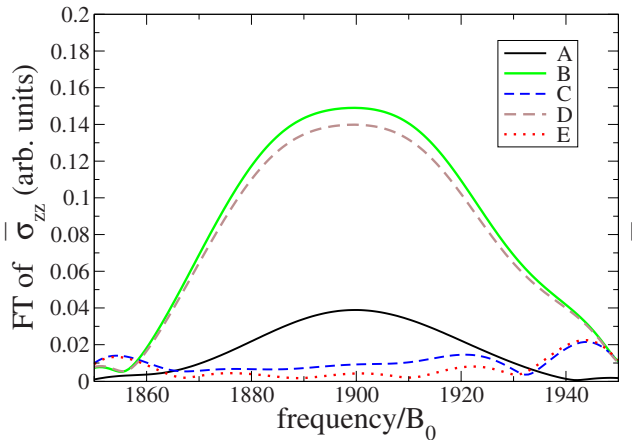

(a)

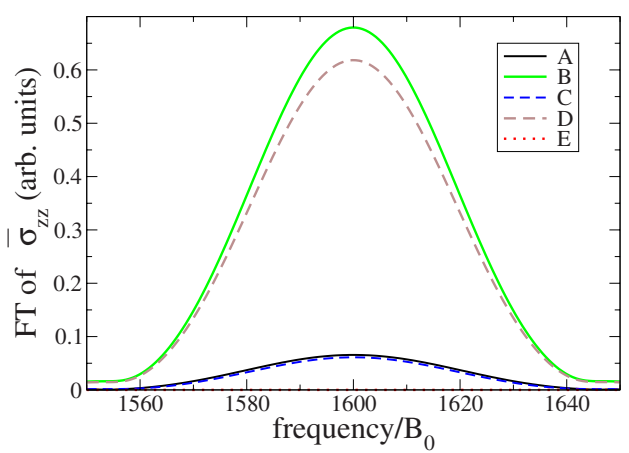

(c)

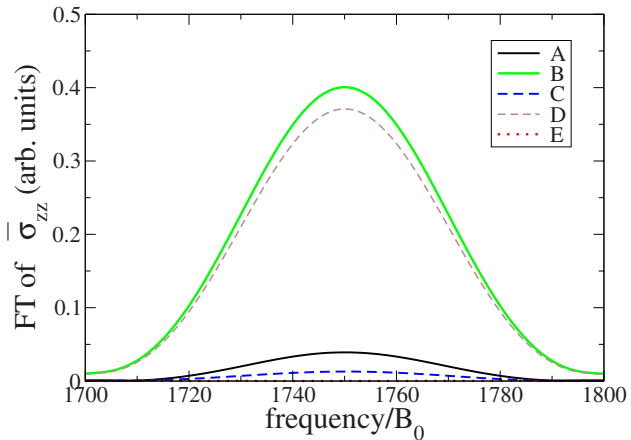

(b)

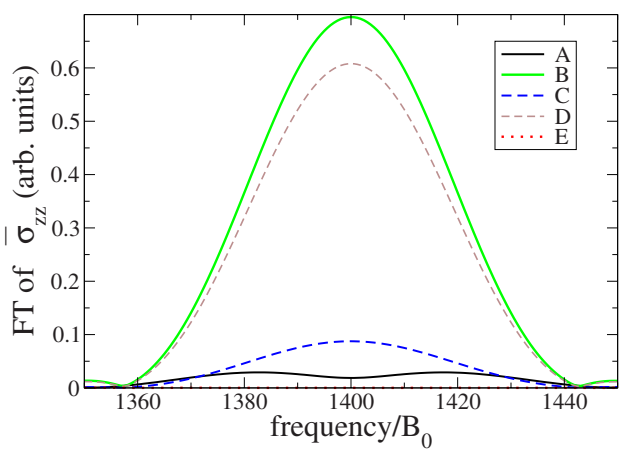

(d)
FIG. 6. (Color online) Figures showing the details of the $\left|f_{2}+f_{1}\right|$ amplitudes of $\left(m_{2} / m_{1}\right)$ values of (a) 0.9 , (b) 0.75 , (c) 0.6 , and (d) 0.4 . 


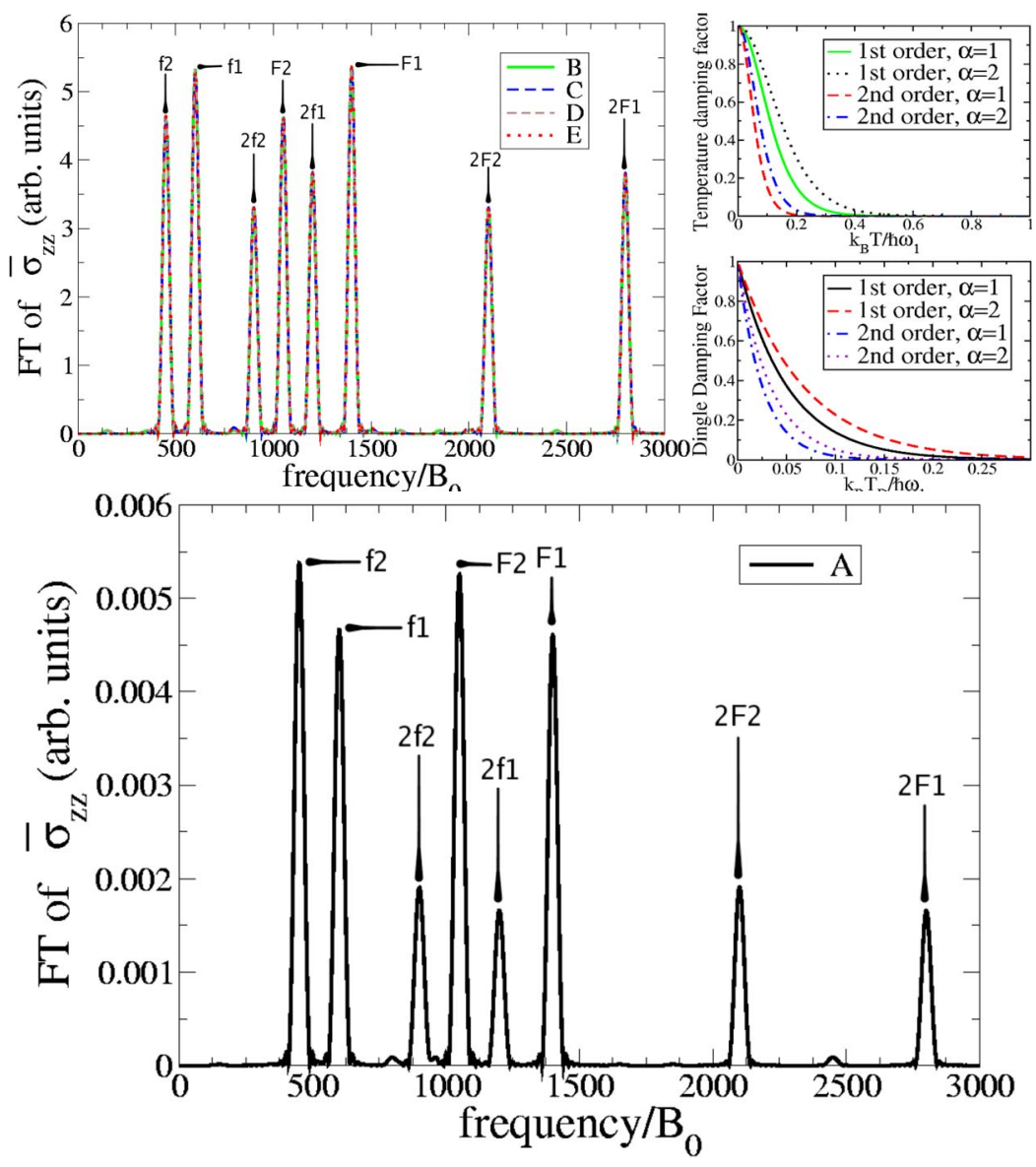

FIG. 7. (Color online) Fourier transforms of the SdH oscillations in the large $t$ limit for a window of $0.9 \leqslant B_{0} / B \leqslant 0.95$ where the frequency is given in terms of $B / B_{0}$ and $\mu=5 t,\left(\omega_{1} / \omega_{2}\right)$ $=\left(m_{2} / m_{1}\right)=0.75, \quad k_{B} T=2.5 \times 10^{-7} t, \quad$ and $B_{0}$ $=m_{1} t / 200 \hbar e$, which is around $4.5 \mathrm{~T}$ when $m_{1}$ $=m_{e}$ and $t=0.1 \mathrm{eV}$, and we have set $T_{D}$ to be zero. The cosine and sine functions in the amplitudes have split the peaks of each harmonic in two; these are labeled $f$ and $F$ corresponding to the neck and belly frequencies, respectively. The $y$ axis in both graphs has been rescaled by a factor of 1000; note that the amplitudes in the case of oscillating $\mu$ alone (A) are much smaller than those of the other cases. The legend in the Fourier transform plots should be interpreted as follows: A-closed system, fixed $\tau$, B-open system, interband scattering; $\mathrm{C}$-closed system, intraband scattering; D-closed system, interband scattering; E-open system, intraband scattering. The plots of the damping factors show how the first and second order damping terms vary with their respective temperatures.

measure of frequency mixing. However, the behavior of the mixing amplitudes are slightly different for each case where they occurred, which may allow the mechanisms involved in experimental systems to be distinguished. In the fourth and fifth cases, we observe frequency mixing due to scattering, which is interesting as we would expect such an effect in the
dHvA oscillations to be virtually negligible (as argued in Sec. III).

\section{ACKNOWLEDGMENT}

The authors would like to thank the EPSRC for funding this work (Grant No. EP/D035589).
${ }^{1}$ D. Shoenberg, Magnetic Oscillations in Metals (Cambridge University Press, Cambridge, 1984).

${ }^{2}$ J. Singleton, Rep. Prog. Phys. 63, 1111 (2000).

${ }^{3}$ M. V. Kartsovnik, Chem. Rev. (Washington, D.C.) 104, 5737 (2004).

${ }^{4}$ N. Doiron-Leyraud, C. Proust, D. LeBoeuf, J. Levallois, J. B. Bonnemaison, R. X. Liang, D. A. Bonn, W. N. Hardy, and L. Taillefer, Nature (London) 447, 565 (2007); A. F. Bangura, J. D. Fletcher, A. Carrington, J. Levallois, M. Nardone, B. Vignolle, P. J. Heard, N. Doiron-Leyraud, D. LeBoeuf, L. Taillefer, S. Adachi, C. Proust, and N. E. Hussey, Phys. Rev. Lett. 100, 047004 (2008); E. A. Yelland, J. Singleton, C. H. Mielke, N. Harrison, F. F. Balakirev, B. Dabrowski, and J. R. Cooper, Phys. Rev. Lett. 100, 047003 (2008).

${ }^{5}$ A. S. Alexandrov and A. M. Bratkovsky, Phys. Rev. Lett. 76, 1308 (1996).
${ }^{6}$ M. Nakano, J. Phys. Soc. Jpn. 66, 19 (1997).

${ }^{7}$ A. S. Alexandrov and A. M. Bratkovsky, Phys. Lett. A 234, 53 (1997).

${ }^{8}$ T. Champel, Phys. Rev. B 65, 153403 (2002); 69, 167402 (2004).

${ }^{9}$ A. S. Alexandrov and A. M. Bratkovsky, Phys. Rev. B 69, 167401 (2004).

${ }^{10}$ K. Kishigi and Y. Hasegawa, Phys. Rev. B 65, 205405 (2002); 72, 045410 (2005).

${ }^{11}$ A. S. Alexandrov and A. M. Bratkovsky, Phys. Rev. B 63, 033105 (2001).

${ }^{12}$ J. Y. Fortin, E. Perez, and A. Audouard, Phys. Rev. B 71, 155101 (2005).

${ }^{13}$ R. A. Shepherd, M. Elliott, W. G. Herrenden-Harker, M. Zervos, P. R. Morris, M. Beck, and M. Ilegems, Phys. Rev. B 60, R11277 (1999). 
${ }^{14}$ E. Ohmichi, Y. Maeno, and T. Ishiguro, J. Phys. Soc. Jpn. 68, 24 (1999).

${ }^{15}$ A. M. Bratkovsky and A. S. Alexandrov, Phys. Rev. B 65 , 035418 (2002).

${ }^{16}$ A. S. Alexandrov and V. V. Kabanov, Phys. Rev. B 76, 233101 (2007).

${ }^{17}$ N. Harrison, J. Caulfield, J. Singleton, P. H. P. Reinders, F. Herlach, W. Hayes, M. Kurmoo, and P. J. Day, J. Phys.: Condens. Matter 8, 5415 (1996); P. S. Sandhu, Ju H. Kim, and J. S. Brooks, Phys. Rev. B 56, 11566 (1997); J. H. Kim, S. Y. Han, and J. S. Brooks, ibid. 60, 3213 (1999); S. Y. Han, J. S. Brooks, and J. H. Kim, Phys. Rev. Lett. 85, 1500 (2000); V. M. Gvozdikov and M. Taut, Phys. Rev. B 75, 155436 (2007); D. Vignolles, A. Audouard, V. N. Laukhin, J. Beard, E. Canadell, N. G. Spitsina, and E. B. Yagubskii, Eur. Phys. J. B 55, 383 (2007).

${ }^{18}$ T. Champel and V. P. Mineev, Phys. Rev. B 66, 195111 (2002); 67, 089901 (2003).
${ }^{19}$ P. D. Grigoriev, Phys. Rev. B 67, 144401 (2003).

${ }^{20}$ T. Champel and V. P. Mineev, Phys. Rev. B 74, 247101 (2006).

${ }^{21}$ R. Kubo, H. Hasegawa, and N. Hashitsume, J. Phys. Soc. Jpn. 14, 56 (1959).

${ }^{22}$ G. D. Mahan, Many-Particle Physics, 2nd ed. (Plenum, New York, 1990).

${ }^{23}$ A. Wasserman and M. Springford, Adv. Phys. 45, 471 (1996).

${ }^{24}$ M. V. Kartsovnik, P. D. Grigoriev, W. Biberacher, N. D. Kushch, and P. Wyder, Phys. Rev. Lett. 89, 126802 (2002).

${ }^{25}$ B. E. Cole, F. M. Peeters, A. Ardavan, S. O. Hill, J. Singleton, W. Batty, J. M. Chamberlain, A. Polisskii, M. Henini, and T. Cheng, J. Phys.: Condens. Matter 9, 3163 (1997).

${ }^{26}$ E. Stade, Fourier Analysis (Wiley, New Jersey, 2005).

${ }^{27}$ J. Y. Fortin and T. Ziman, Phys. Rev. Lett. 80, 3117 (1998); N. Harrison and J. Singleton, ibid. 82, 4148 (1999).

${ }^{28}$ Such as R. Kubo, H. Hasegawa, and N. Hashitsume, Solid State Phys. 17, 279 (1965). 